\title{
Intramedullary spinal sarcoidosis: clinical improvement reflected in T-lymphocyte subpopulation ratios
}

\author{
JS Hawley ${ }^{1}$, JP Ney*,1 and RG Riechers ${ }^{1}$ \\ ${ }^{1}$ Department of Neurology, Walter Reed Army Medical Center, Washington, DC, USA
}

Study design: Case report.

Objectives: A case report of spinal sarcoidosis improving clinically and radiographically with treatment which correlated with improvement in cerebrospinal fluid T-lymphocyte subpopulation ratios.

Setting: Walter Reed Army Medical Center.

Case report: A 46-year-old man presented with an enhancing spinal cord lesion. Lymph node biopsy confirmed sarcoidosis, and cerebrospinal fluid (CSF) analysis showed elevation in the ratio of two T-lymphocyte subpopulations. Treatment with steroids resulted in clinical resolution and immunocytologic improvement in the CSF.

Spinal Cord (2006) 44, 49-51. doi:10.1038/sj.sc.3101782; published online 19 July 2005

Keywords: intramedullary sarcoidosis; spinal sarcoidosis; sarcoidosis lymphocyte subpopulations

\section{Introduction}

Spinal cord disease is a rare and confounding manifestation of sarcoidosis. Cerebrospinal fluid (CSF) markers are insensitive and tissue biopsy carries significant morbidities. ${ }^{1}$ In contrast, pulmonary sarcoidosis has characteristic immunocytologic changes in alveolar fluid. ${ }^{2}$ Small studies suggest an elevated ratio of CSF CD4:CD8 + (T helper:T suppressor or T4:T8) lymphocytes in neurosarcoidosis. ${ }^{3}$ We present a patient with sarcoid myelopathy whose clinical and radiographic diagnosis and treatment response correlated with the CSF immune profile.

\section{Case report}

A 42-year-old man had ascending paresthesias, gait impairment, and erectile dysfunction progressing over 1 month. Neurologic examination revealed absent vibration sense to the iliac crest bilaterally. His gait was widebased, and he had a Romberg sign. Extensor toe signs were present despite normal strength and reflexes.

$\mathrm{He}$ was admitted to the in-patient neurology service. An expansile contrast-enhancing spinal cord lesion

*Correspondence: JP Ney, Department of Neurology, Walter Reed Army Medical Center, 6900 Georgia Avenue, NW, Bldg \#2, Washington, DC 20307, USA

The opinions or assertions contained herein are the private views of the authors and are not to be construed as official or as reflecting the views of the Department of the Army or the Department of Defense was found on a cervical spine MRI (Figure 1a and b). Brain MRI was normal. Electrolytes, complete blood count, B12 panel, and serum angiotensin-converting enzyme (ACE) level were normal. Infectious serologies and PPD were negative. CSF analysis showed a lymphocytic pleocytosis (103 leukocytes $\left./ \mathrm{mm}^{3}\right)$, elevated protein $(93 \mathrm{mg} / \mathrm{dl})$, and undetectable ACE. Flow cytometry demonstrated $63 \%$ of CSF lymphocytes were $\mathrm{T}$ cells and the T4:T8 ratio was elevated at 8.4. Chest CT revealed marked mediastinal lymphadenopathy. Noncaseating granulomas consistent with sarcoidosis were seen on mediastinal lymph node biopsy. His symptoms rapidly improved with high-dose prednisone.

At 2 months after beginning steroid therapy, complaints of erectile dysfunction, parasthesias, and gait imbalance had resolved. Repeat lumbar puncture had three leukocytes $/ \mathrm{mm}^{3}$ with a T4:T8 ratio of 3.0. A second cervical spine MRI showed slight residual contrast uptake (Figure 1c and d).

\section{Discussion}

Sarcoidosis is a granulomatous disease affecting multiple organ systems. Sarcoid granulomas have a core of monocyte-derived cells and an outer layer of CD4 + T lymphocytes. ${ }^{4}$ Active granulomas have representative radiologic findings and immunologic makeup. 

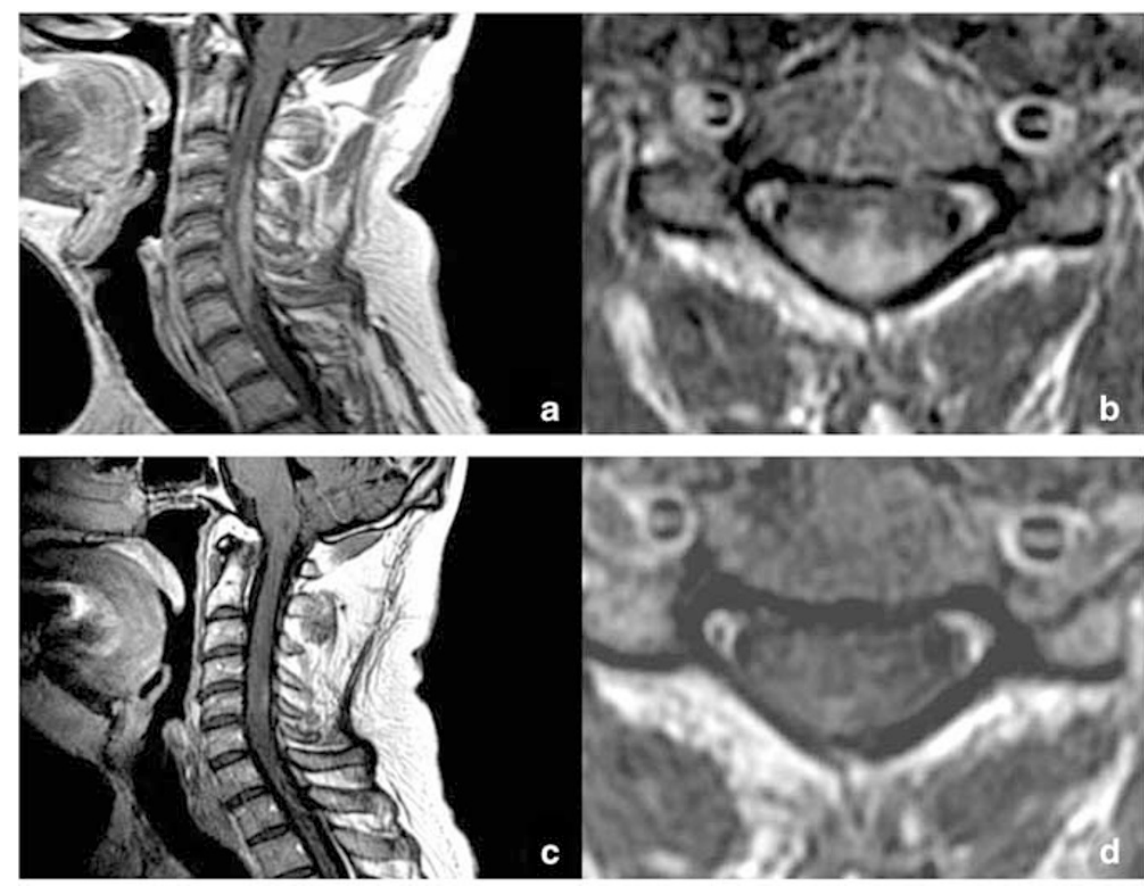

Figure 1 (a) Cervical spine magnetic resonance image (sagittal T1-weighted with contrast) showing cord enlargement and patchy enhancement from $\mathrm{C} 3$ to $\mathrm{C} 7$. (b) Cervical cord magnetic resonance image taken through the level of the mid-C5 vertebral body (axial T1-weighted with contrast) shows the predominant dorsal cord parenchymal and leptomeningeal enhancement. (c, d) Postcontrast T1 images taken 2 months after initiation of steroid treatment

De novo presentation of sarcoid myelopathy confounds an already difficult diagnosis. MRI features are not specific. Fusiform cord enlargement with leptomeningeal spread in the upper cord is typical. Increased T2 signal and heterogenous contrast enhancement is seen. ${ }^{5}$ Fungal or mycobacterial infections and neoplasms such as lymphoma can have similar findings. Elevated CSF protein and lymphocytic pleocytosis are common but not specific. CSF ACE levels are insensitive. ${ }^{6}$ Spinal cord histopathology is the gold standard, but biopsy carries considerable morbidity. Moreover, frozen section may not be reliable. ${ }^{1}$ In our patient, a thorough search for other biopsy sites yielded a diagnosis.

Although mediastinal biopsy was diagnostic in this patient, we saw supportive evidence of sarcoidosis in his CSF T4:T8 ratio. Both alveolar fluid and CSF have immune profiles independent of the systemic circulation. The cytologic makeup of these fluids changes with disease affecting adjacent tissue. Alveolar fluid in active pulmonary sarcoidosis frequently has an elevated T4:T8 ratio in the range of $5-15: 1,{ }^{2}$ reflecting the increased role of T4 lymphocytes in granulomatous diseases. While deep cerebral sarcoid lesions and distal cranial neuropathies often do not show CSF immunocytologic abnormalities, granuloma formation near subarachnoid spaces would have enhanced CSF T4 lymphocyte subpopulation activity. In one series, ${ }^{3}$ only three of eight neurosarcoid patients showed an elevated CSF $\mathrm{T} 4: \mathrm{T} 8$ ratio, yet the ratio was increased in two of three patients with mass lesions. Intramedullary spinal sarcoidosis may be especially prone to elevations in the CSF T4:T8 ratio due to granulomas abutting CSF.

Measures of spinal sarcoidosis disease activity are lacking. Serial MR imaging depicts cord enhancement and enlargement evolving to eventual atrophy without enhancement in later stages. ${ }^{1}$ Imaging in the midst of this process may portray an apparent normalization of the cord despite still-active disease. CSF sampling could provide objective laboratory evidence supporting clinical decline or resolution. In pulmonary sarcoidosis, the T4:T8 ratio of bronchoalveolar fluid mirrors treatment response. ${ }^{2}$ Nonimprovement of the T4:T8 ratio with cyclosporine proved lack of benefit of that drug in pulmonary sarcoid. ${ }^{7}$ In our patient, post-treatment T4:T8 ratio reflected his clinical picture with considerable improvement.

\section{Conclusion}

Further study may elaborate the role of this immunocytologic marker in the diagnosis of spinal sarcoidosis, as a measure of disease activity, and in determining the utility of steroid-sparing immunosuppressants.

\section{References}

1 Junger SS, Stern BK, Levine SR, Sipos E, Marti-Mosso JF. Intramedullary spinal sarcoidosis: clinical and magnetic 
resonance imaging characteristics. Neurology 1993; 43: 333337.

2 Agostini C, Meneghin A, Semenzato G. T-lymphocytes and cytokines in sarcoidosis. Curr Opin Pulm Med 2002; 8: 435440.

3 Stern BJ, Griffin DE, Luke RA, Krumholz A, Johns CJ. Neurosarcoidosis: cerebrospinal fluid lymphocyte subpopulations. Neurology 1987; 37: 878-881.

4 Agostini C, Basso U, Semenzato G. Cells and molecules involved in the development of sarcoid granuloma. J Clin Immunol 1998; 18: 184-192.
5 Smith JK, Matheus MG, Castillo M. Imaging manifestations of neurosarcoidosis. Am J Radiol 2004; 182: 289-295.

6 Tahmoush AJ et al. CSF-ACE activity in probable CNS neurosarcoidosis. Sarcoidosis Vasc Diffuse Lung Dis 2002; 19: 191-197.

7 Martinet Y, Pinkston P, Saltini C, Spurzem J, MullerQuernheim J, Crystal RG. Evaluation of the in vitro and in vivo effects of cyclosporine on the lung T-lymphocyte alveolitis of active pulmonary sarcoidosis. Am Rev Resp Dis 1988; 138: 1242-1248. 